\title{
Temperature dependence of the generalized vibrational density of states of sodium bismuth titanate in the ferroelectric phase
}

\author{
Authors: S. Lushnikov, S. Gvasaliya, I. Siny, I. \\ Sashin, V. Hugo Schmidt, and T. Uesu
}

NOTICE: this is the author's version of a work that was accepted for publication in Solid State Communications. Changes resulting from the publishing process, such as peer review, editing, corrections, structural formatting, and other quality control mechanisms may not be reflected in this document. Changes may have been made to this work since it was submitted for publication. A definitive version was subsequently published in Solid State Communications [VOL\# 116, ISSUE\# 1, (2000)] DOI\# 10.1016/S0038-1098(00)00265-9

S. Lushnikov, S. Gvasaliya, I. Siny, I. Sashin, V.H. Schmidt, and T. Uesu, "Temperature dependence of the generalized vibrational density of states of sodium bismuth titanate in the ferroelectric phase," Solid State Communications 116 (1), 41-45 (2000). http://dx.doi.org/10.1016/S0038-1098(00)00265-9

Made available through Montana State University's $\underline{\text { ScholarWorks }}$ scholarworks. montana.edu 


\title{
Temperature dependence of the generalized vibrational density of states of sodium bismuth titanate in the ferroelectric phase
}

\author{
S.G. Lushnikov ${ }^{\mathrm{a}, *}$, S.N. Gvasaliya ${ }^{\mathrm{a}}$, I.G. Siny ${ }^{\mathrm{a}}$, I.L. Sashin ${ }^{\mathrm{b}}$, V.H. Schmidt ${ }^{\mathrm{c}}$, Y. Uesu $^{\mathrm{d}}$ \\ ${ }^{a}$ A.F. Ioffe Physical Technical Institute, 194021 St. Petersburg, Russian Federation \\ ${ }^{\mathrm{b}}$ Frank Laboratory of Neutron Physics, Joint Institute for Nuclear Research, 141980 Dubna, Russian Federation \\ ${ }^{\mathrm{c}}$ Physics Department, Montana State University, Bozeman, MT 59717, USA \\ ${ }^{\mathrm{d}}$ School of Science and Engineering, Waseda University, Shinjuku-ku, Tokyo 160, Japan
}

Received 14 June 2000; accepted 21 June 2000 by E.L. Ivchenko

\begin{abstract}
This paper describes studies of relaxor ferroelectric $\mathrm{Na}_{1 / 2} \mathrm{Bi}_{1 / 2} \mathrm{TiO}_{3}(\mathrm{NBT})$ by inelastic incoherent neutron scattering at temperatures from 50 to $300 \mathrm{~K}$. Experimental time-of-flight spectra were recalculated into the generalized vibrational density of states (VDOS) function in terms of one-particle incoherent approximation and compared with the behavior of Raman spectra. It is shown that the VDOS of NBT in the ferroelectric phase has nontrivial temperature dependence. The relation between the temperature dependence of VDOS and the instability of the NBT crystalline lattice below room temperature is discussed. (C) 2000 Published by Elsevier Science Ltd.
\end{abstract}

Keywords: A. Ferroelectrics; D. Anharmonicity; D. Phonons

PACS: 77.80; 61.12.-q; 64.60; 78.30; 63.50.+x; 63.20; 77.84.Dy

\section{Introduction}

The majority of relaxor ferroelectrics (relaxors) known at present belong to perovskite-like compounds with the common formula $\mathrm{AB}^{\prime}{ }_{x} \mathrm{~B}^{\prime \prime}{ }_{1-x} \mathrm{O}_{3}$ disordered with respect to the B sublattice. Sodium bismuth titanate $\mathrm{Na}_{1 / 2} \mathrm{Bi}_{1 / 2} \mathrm{TiO}_{3}$ (NBT) is among the group of relaxor ferroelectrics with the common formula $\mathrm{A}_{x}^{\prime} \mathrm{A}^{\prime \prime}{ }_{1-x} \mathrm{BO}_{3}$ [1]. According to $\mathrm{X}$-ray and neutron structural studies, at room temperature it has symmetry R3c [2-4]. The rhombohedral symmetry results from the following sequence of phase transitions: cubic phase $\stackrel{T_{\mathrm{cl}}=820 \mathrm{~K}}{\longrightarrow}$ tetragonal phase $\stackrel{T_{\mathrm{c} 2}=593 \mathrm{~K}}{\longrightarrow}$ rhombohedral phase. At $T_{\mathrm{c} 3}=500 \mathrm{~K}$ a ferroelectric state arises in an NBT crystal [5]. However, X-ray diffraction studies have shown that deviations from an ideal cubic structure are extremely small and depend on the sample quality [6]. In many respects the behavior of the NBT crystal remains pseudocubic at temperatures below $T_{\mathrm{c} 1}$ as well. Elastic properties of NBT are well described in terms of cubic symmetry [7], and there is a striking similarity between acoustic anomalies in NBT and the model relaxor $\mathrm{PbMg}_{1 /}$ ${ }_{3} \mathrm{Nb}_{2 / 3} \mathrm{O}_{3}$ (PMN) [8]. Considerable contributions to determination of the sequence of phase transitions in NBT were made by neutron scattering [3,9] and Raman light scattering [10-12] studies of its lattice dynamics. Of great importance for understanding behavior of relaxors were similarities between the relaxor ferroelectrics PMN and NBT [8]. Nevertheless, the lattice dynamics of both NBT and PMN in the ferroelectric phase after onset of macroscopic polarization still remains unclear. The primary subject of great controversy is the nature of the lowtemperature phase in these relaxors because the suggested models, such as glass-like phase [13], nanodomain [14], spherical random bond random field [15] and some other models, do not comprehensively describe the whole body of experimental evidence. For this reason it appeared reasonable to continue investigations of the NBT lattice dynamics by inelastic neutron scattering and to compare the obtained results with the data on the PMN vibrational spectrum [16-18].

The goal of this work was to study the behavior of the vibrational spectrum of NBT below room temperature by 


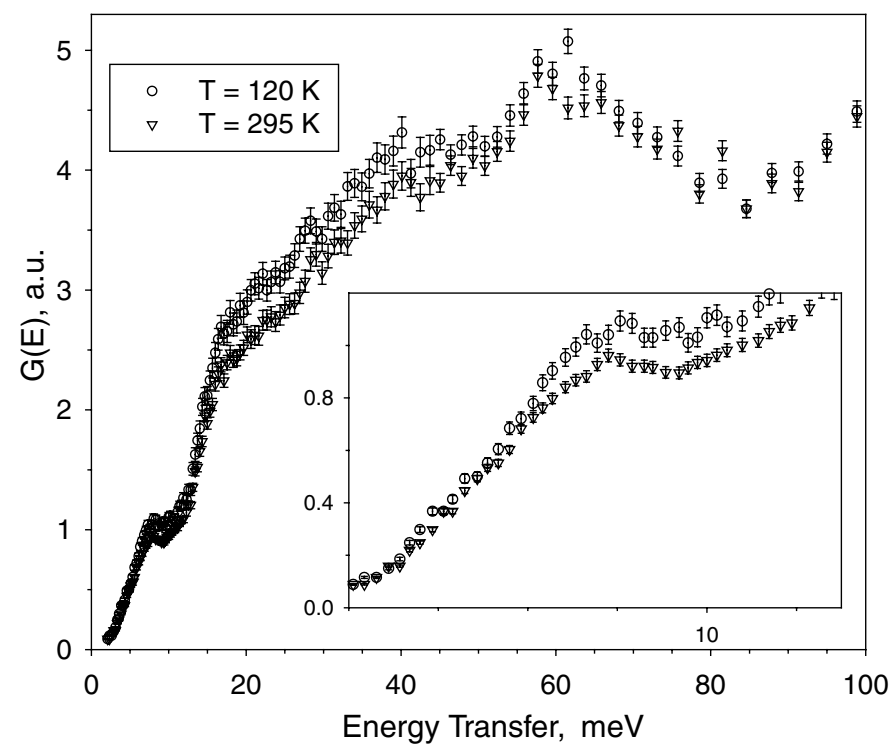

Fig. 1. Generalized density of states function VDOS of relaxor ferroelectric NBT at 290 and $120 \mathrm{~K}$. The inset shows the low-frequency part of VDOS on an enlarged scale.

inelastic incoherent neutron scattering and compare the results with the Raman data.

\section{Experimental}

Inelastic neutron scattering (INS) experiments were performed with the KDSOG-M inverse geometry spectrometer installed at the IBR-2 pulsed high-flux reactor of the Joint Institute for Nuclear Research in Dubna. The INS spectra were recorded in the same fashion for eight scattering angles in the range from 30 to $140^{\circ}$ in the neutron energy loss regime. This allowed the lattice dynamics of the samples to be studied at low temperatures. The neutron energy loss was measured by an analyzer consisting of a polycrystalline beryllium filter cooled to liquid nitrogen temperature and single crystals of pyrolytic graphite. The sample was sodium bismuth titanate $\left(\mathrm{Na}_{1 / 2} \mathrm{Bi}_{1 / 2} \mathrm{TiO}_{3}\right)$ powder with a weight of $120 \mathrm{~g}$.

Inelastic neutron scattering was measured at temperatures from 50 to $295 \mathrm{~K}$. Spectra were recorded at $T=50,120$, and $295 \mathrm{~K}$. The signal accumulation time varied from 10 to $26 \mathrm{~h}$. The measured time-of-flight spectra were normalized by intensity to a 10-h exposure, the background from the cryostat and sample holder being subtracted. The obtained data were recalculated to the generalized vibrational density of states $G(\omega)$ in the incoherent one-particle approximation as

$$
\left.G(\omega)=\frac{\omega}{n(\omega)+1} \frac{\mathbf{k}_{0}}{\mathbf{k}_{1}} \frac{1}{\mathbf{Q}^{2}} \frac{\mathrm{d}^{2} \sigma}{\mathrm{d} \Omega \mathrm{d} E^{\prime}}\right)_{\text {incoh }}
$$

where $\mathbf{k}_{0}$ and $\mathbf{k}_{1}$ are the neutron wave vectors prior and after scattering from the sample, respectively, $\mathbf{Q}=\mathbf{k}_{1}-\mathbf{k}_{0} ; E^{\prime}$ the scattered neutron energy, $\left(\left(\mathrm{d}^{2} \sigma\right) /\left(\mathrm{d} \Omega \mathrm{d} E^{\prime}\right)\right)_{\text {incoh }}$ the partial differential cross section for incoherent scattering, and $n(\omega)$ is the Bose-Einstein factor. Multiple and manyparticle processes were ignored.

\section{Results and discussion}

\subsection{Inelastic incoherent neutron scattering}

Fig. 1 shows generalized vibrational density of states of NBT (the low-energy region of the density of states is demonstrated in the inset on a larger scale). Let us consider the $G(E)$ function from the point of view of specific features of the NBT structure. It is known that the low-frequency region of the vibrational density of state is sensitive to disorder. This fact is widely used in studies and comparative analysis of systems with different types of disorder, such as crystals, orientational glasses, canonical glasses, and amorphous polymers [19-21]. A number of authors have shown that disorder exists in the NBT structure when regions with local ordering of $1: 1 \mathrm{Na}^{+}$and $\mathrm{Bi}^{3+}$ cations with sizes of the order of $2 \mathrm{~nm}$ are present $[5,11,12]$. In PMN such regions with ordering of $1: 2 \mathrm{Mg}^{2+}$ and $\mathrm{Nb}^{5+}$ ions were identified by different methods [8]. Their presence manifested itself in the vibrational spectrum as specific excitations with fracton spectral dimension in the low-frequency region of the density of state function [17,18]. Analysis of the lowfrequency region of the $G(E)$ function of NBT has not revealed excitations with fracton spectral dimensions that could be compared with nanoregions, as was done for PMN. 


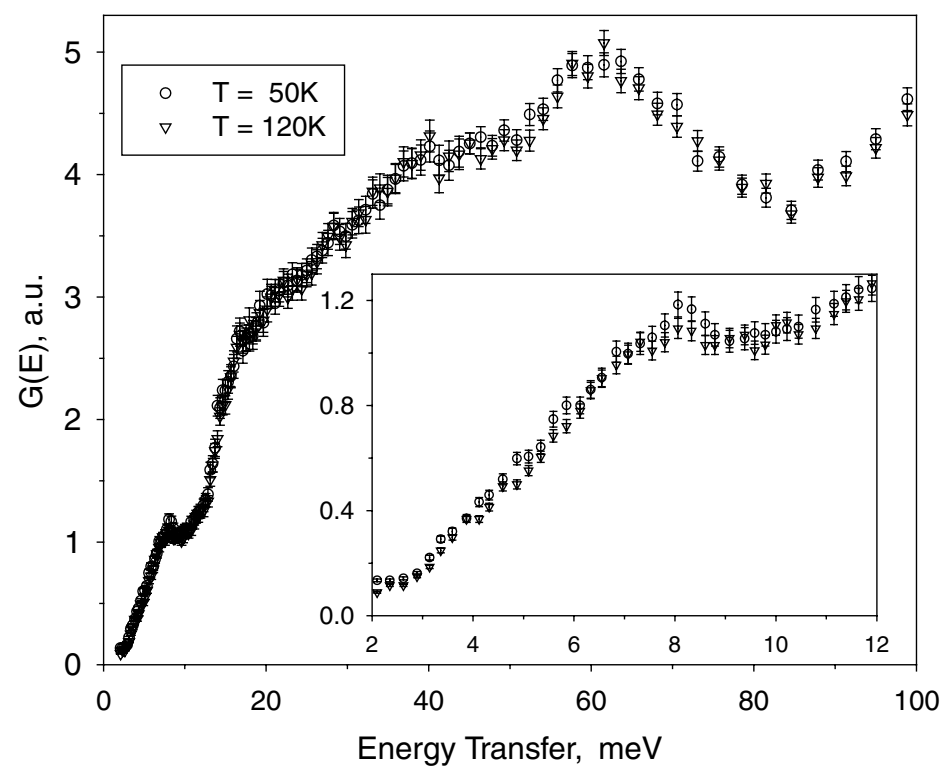

Fig. 2. Generalized density of states function VDOS of relaxor ferroelectric NBT at 120 and $50 \mathrm{~K}$. The error is shown by bars. The inset shows the low-frequency part of VDOS on an enlarged scale.

It is easily seen from Fig. 1 that decreasing temperature gives rise to changes in the $G(E)$ function in the region of transferred energies $4-55 \mathrm{meV}$. The vibrational density of states (VDOS) of NBT increases as temperature is lowered to $120 \mathrm{~K}$. The changes in the $2-4 \mathrm{meV}$ region are small and do not exceed experimental error. Discussion of the highfrequency region above $55 \mathrm{meV}$ where the multiphonon contribution increases is beyond the scope of this paper. Note that according to expression (1) the trivial temperature dependence is excluded from consideration, and the observed evolution of the VDOS of NBT is not associated with the Bose-Einstein population factor as temperature varies from 295 to $120 \mathrm{~K}$. A decrease in temperature from 120 to $50 \mathrm{~K}$ does not lead to a significant variation in $G(E)$ (Fig. 2) in either the low-frequency or the high-frequency energy region, within experimental error. This temperature dependence of $G(E)$ in NBT is anomalous and is not likely to be associated with either the anharmonicity responsible for VDOS evolution in fused glass $\mathrm{B}_{2} \mathrm{O}_{3}$ [22] or with the contribution of structural relaxation observed in metallic glasses $[23,24]$. Therefore, it is reasonable to invoke data obtained by other techniques and to see whether they correlate with the behavior of the relaxor ferroelectric NBT phonon subsystem observed by inelastic neutron scattering.

\subsection{Light scattering and soft mode}

Light scattering in NBT crystals was studied in Refs. [10-12]. The hypothesis of Fm3m symmetry for the paraphase, which allowed correct description of the scattering spectra [11,12], was later confirmed [6]. In this paper we discuss the low-frequency region of the Raman spectra. Single crystals of NBT grown by the Czochralski technique were used for the experiments. Light scattering spectra were excited by an argon ion laser with $\lambda=514.5 \mathrm{~nm}$. A cubic sample with edges of about $5 \mathrm{~mm}$ was illuminated along [001], and right-angle spectra were analyzed by a Cary- 82 triple spectrophotometer.

The low-frequency mode is well seen in the Raman spectra below room temperature (Fig. 3a). This mode obviously softens when temperature increases. However, it is impossible to follow the mode frequency at higher temperatures, especially in the range of phase transitions. The mode becomes a shoulder in the low-frequency part of the spectrum and then merges with the growing Rayleigh wing. Fig. 3b demonstrates our attempt to consider this mode as a soft mode obeying a usual law $(\Delta \nu)^{2} \propto\left(T_{0}-T\right)$. The extrapolation of $(\Delta \nu)^{2}(T)$ to higher temperatures yields $T_{0} \sim 510 \mathrm{~K}$, which is close to $T_{\mathrm{c} 3}$. It seems that at the initial stage the phase transition is driven by the lattice instability related to vibrational modes. In this respect our data agree with the neutron scattering studies by a triple-axis spectrometer where the soft mode at the Brillouin boundary was also found far from the phase transition range to the cubic phase [3]. When the phase transition from the cubic to tetragonal state was approached from above, this mode became overdamped, so the integral scattering intensity had to be analyzed in order to reveal true softening.

Comparison of the behavior of the soft mode and changes in $G(E)$ of relaxor ferroelectric NBT in the temperature 

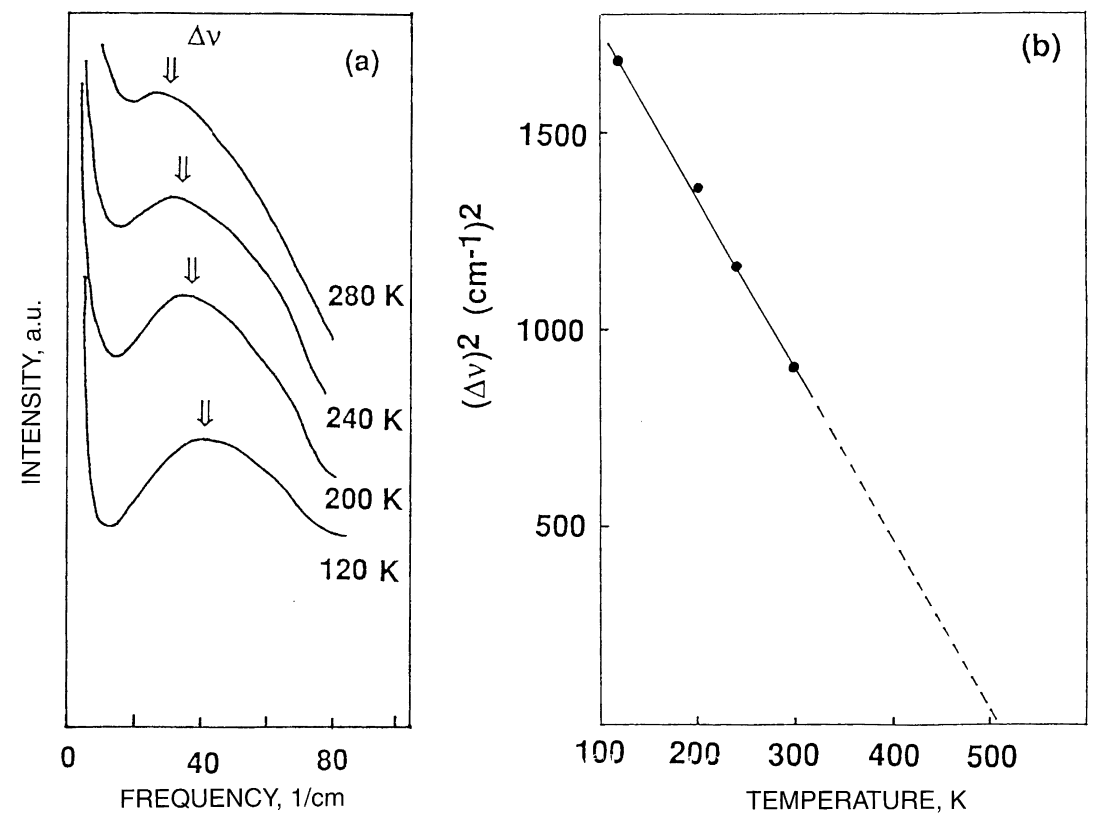

Fig. 3. (a) The low-frequency part of Raman spectra with mode softening on heating. (b) The temperature dependence of $(\Delta \nu)^{2}$ for this mode.

range $290-120 \mathrm{~K}$ has revealed an obvious correlation between them. Therefore it can be supposed that the anomalous behavior of the density of states is due to the instability of the NBT crystalline lattice. In inelastic light scattering experiments this instability manifests itself as a soft-mode behavior of the optical phonon and as "softening" of the hypersonic acoustic phonon, while in inelastic neutron scattering it shows up as a nontrivial temperature dependence of $G(E)$. Probably, all these features can be regarded as the result of processes beginning in the hightemperature region and completed in the vicinity of $100 \mathrm{~K}$. This supposition is supported by the absence of changes in $G(E)$ at temperatures from 120 to $50 \mathrm{~K}$ (Fig.2b). In our opinion, a fairly sharp change in the behavior of the density of states in the NBT crystal can be caused primarily by two factors: a change in the pattern of the soft-mode behavior or termination of the interaction competition effect of the order parameters at the $\Sigma$ line connecting the $\mathrm{R}$ and $\mathrm{M}$ points of the Brillouin zone where high-temperature phase-transitions are realized.

\subsection{Similarity in the behavior of VDOS of relaxor ferroelectrics PMN and NBT}

The temperature dependence of $G(E)$ of relaxor ferroelectric NBT is similar in many respects to the behavior of the density of states of another relaxor ferroelectric PMN in the ferroelectric phase [16]. In both cases major changes in $G(E)$ occur in the ranges 5-10 and 18$40 \mathrm{meV}$. In all cases an increase in the density of states in the energy ranges indicated above occurs with decreasing temperature. However, the temperature behavior of the vibrational density of states of NBT exhibits an important feature (maybe the feature of principal importance): as temperature decreases from 120 to $50 \mathrm{~K}, G(E)$ no longer changes, and the observed differences are within the experimental error limits (see Fig. 2), while the density of states of PMN varies in the whole temperature range studied (30-290 K). A direct correlation between the behavior of the generalized density of states function and phonon subsystem are observed for both PMN and NBT.

In Ref. [16] the temperature dependence of $G(E)$ in the ferroelectric phase of PMN was attributed to the instability of the crystalline lattice when a structural phase transition in the vicinity of $200 \mathrm{~K}$ occurring in an external electric field is frustrated. Temperature changes in $G(E)$ reveal the "hidden" dynamics of the PMN crystalline lattice. The "hidden" dynamics also clearly manifests itself (in the form of anomalies in the temperature dependences near $200 \mathrm{~K}$ ) in the behavior of the relaxation mode found in the light scattering spectra [25] and in damping of hypersonic acoustic phonons [26,27]. In NBT, the crystalline lattice dynamics clearly manifests itself in the entire temperature range studied by softening of the low-frequency optical phonon. Thus it can be supposed that the crystalline lattice dynamics in the ferroelectric phase of relaxor ferroelectrics PMN and NBT has anomalies giving rise to changes in the density of states function.

The present study does not completely elucidate these phenomena. Additional neutron scattering experiments should be carried out and other techniques should also be invoked. 


\section{Conclusions}

1. Analysis of the low-energy region of the generalized density of states function of the relaxor ferroelectric NBT has not revealed any excitations with the parameters characteristic of fractal objects.

2. A decrease in temperature has been found to lead to changes in the generalized density of states. In our opinion, this is the consequence of instability of the crystalline lattice of NBT in the ferroelectric phase. This instability manifests itself also in the soft-mode behavior of the low-lying mode in Raman spectra of NBT.

3. Comparison of the temperature variations of the generalized density of states of NBT and PMN has shown that $G(E)$ is no doubt sensitive to the "hidden" dynamics of the crystalline lattice associated, among other things, with a suppressed phase transition.

\section{Acknowledgements}

The authors would like to thank T.A. Shaplygina and N.I. Zaytseva for preparing and testing the samples. The work was supported by Russian Foundation for Basic Research, grant 99-02-18316 and NSF Grant DMR-9805272. One of the authors (SNG) was supported by an ISSEP grant.

\section{References}

[1] M.E. Lines, A.M. Glass, Principles and Applications of Ferroelectrics and Related Materials, Clarendon Press, Oxford, 1977.

[2] J.A. Zvirgzds, P.P. Kapostins, J.V. Zvirgzde, T.V. Kruzina, Ferroelectrics 40 (1982) 75.

[3] S.B. Vakhrushev, B.E. Kvyatkovsky, R.S. Malysheva, N.M. Okuneva, E.L. Plachenova, P.P. Syrnikov, Kristallografiya 34 (1989) 154 [Sov. Phys.-Crystallogr. 34 (1989) 89].

[4] S.-E. Park, K.S. Hong, J. Appl. Phys. 79 (1996) 383.

[5] C.-S. Tu, I.G. Siny, V.H. Schmidt, Phys. Rev. B 49 (1994) 11550.
[6] S.-E. Park, S.-J. Chung, I.-T. Kim, K.S. Hong, J. Am. Ceram. Soc. 77 (1994) 2641.

[7] I.G. Siny, C.-S. Tu, V.H. Schmidt, Phys. Rev. B 51 (1995) 5659.

[8] I.G. Siny, S.G. Lushnikov, R.S. Katiyar, V.H. Schmidt, Ferroelectrics 226 (1999) 191.

[9] S.B. Vakhrushev, V.A. Isupov, B.E. Kvyatkovsky, N.M. Okunrva, I.P. Pronin, G.A. Smolensky, P.P. Syrnikov, Ferroelectrics 63 (1985) 153.

[10] M.-S. Zhang, J.F. Scott, J.A. Zvirgzds, Ferroelect. Lett. 6 (1986) 147.

[11] I.G. Siny, T.A. Smirnova, T.V. Kruzina, Fiz. Tverd. Tela 33 (1991) 110 [Sov. Phys.-Solid State 33 (1991) 61].

[12] I.G. Siny, T.A. Smirnova, T.V. Kruzina, Ferroelectrics 124 (1991) 207.

[13] D. Viehland, S.J. Jang, L.E. Cross, J. Appl. Phys. 68 (1990) 6.

[14] V. Westphal, W. Kleemann, M.D. Glinchuk, Phys. Rev. Lett. 68 (1992) 847.

[15] R. Pirc, R. Blinc, Phys. Rev. B 60 (1999) 13470.

[16] S.G. Lushnikov, S.N. Gvasaliya, I.G. Siny, E.A. Goremychkin, I.L. Sashin, Ferroelectrics 226 (1999) 147.

[17] S.G. Lushnikov, S.N. Gvasaliya, I.G. Siny, Physica B (Amsterdam), 263/264 264 (1999) 286.

[18] S.N. Gvasaliya, S.G. Lushnikov, I.G. Siny, I.L. Sashin, Kristallografiya 44 (1999) 284 [Crystallography Reports 44 (1999) 250].

[19] L.M. Needham, M. Cutroni, A.J. Dianoux, H.M. Rosenberg, J. Phys.: Condens. Matter 5 (1993) 637.

[20] M.A. Ramos, S. Vieira, F.J. Bermejo, J. Davidowski, H.E. Fisher, H. Schober, M.A. Gonzalez, C.K. Loong, D.L. Price, Phys. Rev. Lett. 78 (1997) 82.

[21] F.J. Bermejo, G.J. Cuello, J. Davidowski, A. Criado, H.E. Fisher, H. Schober, M.A. Gonzalez, S.M. Bennington, Physica B (Amsterdam) 241-243 (1998) 883.

[22] R. Fernandez-Perea, F.J. Bermejo, A. de Bernabe, C.-K. Loong, Phys. Lett. A 234 (1997) 169.

[23] J.-B. Suck, J. Non-Cryst. Solids 205-207 (1996) 592.

[24] F. Dugain, M. Mihalkovic, J.-B. Suck, Mater. Sci. Engng A 226-228 (1997) 967.

[25] I.G. Siny, S.G. Lushnikov, R.S. Katiyar, E.A. Rogacheva, Phys. Rev. B 56 (1997) 7962.

[26] R. Laiho, S.G. Lushnikov, S.D. Prokhorova, I.G. Siny, Fiz. Tverd. Tela 32 (1990) 3490 [Soviet Phys.-Solid State 32 (1990) 2024].

[27] R. Laiho, S.G. Lushnikov, S.D. Prokhorova, I.G. Siny, Ferroelectrics 125 (1992) 493. 